\title{
BIODEGRADAÇÃO DE FENOL POR UMA NOVA LINHAGEM DE Aspergillus sp. ISOLADA DE UM SOLO CONTAMINADO DO SUL DO BRASIL
}

\author{
Cátia Tavares dos Passos, Janaína Fernandes de Medeiros Burkert, Susana Juliano Kalil e Carlos André Veiga Burkert* \\ Escola de Química e Alimentos, Universidade Federal do Rio Grande, CP 474, 96201-900 Rio Grande - RS, Brasil
}

Recebido em 6/6/08; aceito em 24/10/08; publicado na web em 12/2/09

\begin{abstract}
BIODEGRADATION OF PHENOL BY A NEWLY Aspergillus sp. STRAIN ISOLATED FROM A CONTAMINATED SOIL IN SOUTHERN BRAZIL. The main goal of this work was to study the biodegradation of phenol in batch mode by a filamentous fungus isolated from a contaminated site in Southern Brazil. A better performance was obtained by previous adaptation of the microorganism to the toxic chemical. A $2^{3}$ experimental design was proposed and it could be observed total phenol degradation in $72 \mathrm{~h}$ using 500 $\mathrm{mg} \mathrm{L}^{-1}$ glucose, inoculum of $20 \%$ and agitation of $200 \mathrm{rpm}$, resulting a biodegradation rate of $3.76 \mathrm{mg} \mathrm{L}^{-1} \mathrm{~h}^{-1}$. In relation to phenol tolerance, Aspergillus sp. LEBM2 was able to consume up to $989 \pm 15 \mathrm{mg} \mathrm{L}^{-1}$.
\end{abstract}

Keywords: bioremediation; filamentous fungi; phenol.

\section{INTRODUÇÃO}

Fenol e seus derivados constituem uma importante classe de contaminantes ambientais pela sua presença em muitos efluentes industriais, incluindo refinarias, plantas petroquímicas, siderúrgicas, indústrias de cerâmicas e de resinas fenólicas, entre outros. ${ }^{1}$ Embora sendo biodegradável tanto por via aeróbia como anaeróbia, o fenol é tóxico para a maioria dos microrganismos, principalmente não aclimatados, em concentração de apenas $10 \mathrm{mg} \mathrm{L}^{-1}$, podendo ser inibidor do crescimento mesmo para as espécies que o utilizam como substrato, causando problemas em estações de tratamento de efluentes. ${ }^{2}$ A resolução CONAMA n ${ }^{\circ} 357$, publicada em 18 de março de 2005, define como padrão de lançamento para efluentes industriais o teor de $0,5 \mathrm{mg} \mathrm{L}^{-1}$ de fenóis totais, ${ }^{3}$ valor considerado baixo frente às concentrações geradas pelas indústrias. Portanto, a remoção eficiente de fenol de efluentes industriais é de grande importância para a proteção ambiental e novas estratégias têm sido propostas para atingir esta finalidade.

A bioaumentação por microrganismos autóctones selecionados constitui uma excelente alternativa para a biodegradação do fenol. Diversos autores têm mencionado o uso de microrganismos visando a remoção de fenol em efluentes industriais como, por exemplo, Penicillium, ${ }^{1}$ Aspergillus,${ }^{1,4}$ Graphium,${ }^{1,5}$ Candida,${ }^{6,7}$ Pseudomonas,${ }^{8}$ Acinetobacter $^{9}$ e Rhodococcus. ${ }^{10}$ No entanto, a prospecção de novas linhagens degradadoras, adaptadas a condições ambientais locais, e sua caracterização quanto à capacidade biodegradativa e tolerância à toxidade constituem aspectos importantes a serem ainda explorados. Para a avaliação da toxicidade e biodegradabilidade, os ensaios em batelada são úteis para classificar variáveis importantes, verificando seus efeitos no desempenho do microrganismo no processo biodegradativo.

O objetivo deste trabalho foi avaliar a biodegradação do fenol por Aspergillus sp. LEBM2, verificando-se a influência da adaptação prévia do fungo, os efeitos dos parâmetros de cultivo em batelada e a tolerância ao fenol.

\footnotetext{
*e-mail: burkert@ vetorial.net
}

\section{PARTE EXPERIMENTAL}

\section{Água residuária sintética}

Os constituintes da água residuária sintética foram preparados na forma de soluções concentradas, sendo esterilizadas separadamente em autoclave (soluções de minerais e de glicose) e por filtração utilizando membrana Millipore de 0,22 $\mu \mathrm{m}$ (solução de fenol). Após as soluções foram misturadas, de forma a resultar na seguinte composição no início dos cultivos ( $\left.\mathrm{mg} \mathrm{L}^{-1}\right)$ : 400, $\mathrm{KH}_{2} \mathrm{PO}_{4} ; 200, \mathrm{MgSO}_{4} .7 \mathrm{H}_{2} \mathrm{O}$; $100, \mathrm{NaCl} ; 25, \mathrm{CaCl}_{2} \cdot 2 \mathrm{H}_{2} \mathrm{O} ; 3, \mathrm{MnSO}_{4} \cdot \mathrm{H}_{2} \mathrm{O} ; 500, \mathrm{NH}_{4} \mathrm{NO}_{3} \cdot \mathrm{H}_{2} \mathrm{O}$; glicose e fenol em concentrações variáveis.

\section{Microrganismo}

Utilizou-se o fungo Aspergillus sp. LEBM2, isolado de um solo contaminado por hidrocarbonetos derivados de petróleo, ${ }^{11}$ na região da cidade do Rio Grande, RS, Brasil, sendo mantido a $4{ }^{\circ} \mathrm{C}$, em ágar batata dextrose (PDA).

\section{Influência da adaptação sobre a capacidade biodegradativa}

Para obtenção dos diferentes inóculos, empregaram-se frascos erlenmeyer de $500 \mathrm{~mL}$ onde foram adicionados $150 \mathrm{~mL}$ de água residuária sintética contendo diferentes fontes de carbono: somente fenol (250 mg L$\left.{ }^{-1}\right)$, glicose $\left(250 \mathrm{mg} \mathrm{L}^{-1}\right)$ e fenol $\left(250 \mathrm{mg} \mathrm{L}^{-1}\right)$ e somente glicose $\left(250 \mathrm{mg} \mathrm{L}^{-1}\right)$.

Cada um destes frascos recebeu três unidades de $1 \mathrm{~cm}$ de diâmetro, provenientes do cultivo prévio do fungo em placas de Petri com PDA (sem adição de fenol) durante 5 dias a $25^{\circ} \mathrm{C} . .^{12}$

Após incubação a $25^{\circ} \mathrm{C}$ por 7 dias, os diferentes inóculos obtidos foram transferidos para frascos erlenmeyers de $500 \mathrm{~mL}$ contendo água residuária sintética acrescida de $250 \mathrm{mg} \mathrm{L}^{-1}$ de fenol como única fonte de carbono. $\mathrm{O}$ volume de inóculo correspondeu a $10 \%$ do volume total, de $150 \mathrm{~mL}$. Paralelamente foi realizado um ensaio controle, sem a inoculação do microrganismo, para avaliar as perdas abióticas. Os experimentos foram realizados em triplicata, de forma estática (sem agitação), a $25^{\circ} \mathrm{C}$.

Os resultados foram avaliados pela taxa de biodegradação de fenol (em mg L $\mathrm{L}^{-1} \mathrm{~h}^{-1}$ ), obtida pelo acompanhamento da concentração 
do fenol ao longo do tempo, sendo esta determinada nas amostras previamente filtradas e diluídas, através do método baseado no uso do reagente de Folin-Ciocalteau, ${ }^{5}$ com posterior leitura de absorbância a $750 \mathrm{~nm}$, utilizando uma curva de calibração de fenol na faixa de 0,25 a $10 \mathrm{mg} \mathrm{L}^{-1}$.

\section{Influência dos parâmetros de cultivo na biodegradação do fenol}

Efetuou-se um planejamento fatorial completo envolvendo 3 variáveis ( $2^{3}$ ensaios mais três pontos centrais), visando aumentar a eficiência de biodegradação do fenol por Aspergillus sp. LEBM2. As variáveis estudadas foram: concentração de glicose $(0$ a $500 \mathrm{mg}$ $\left.\mathrm{L}^{-1}\right)$, agitação (0 a $200 \mathrm{rpm}$ ) e volume de inóculo (10 a 20\%). Nos experimentos, foram acompanhadas as concentrações de fenol por Folin-Ciocateau ${ }^{5}$ e de glicose pelo método $3,5 \mathrm{DNS}^{13}{ }^{13}$ e a resposta analisada foi a taxa de biodegradação de fenol.

O inóculo foi preparado conforme estabelecido na etapa anterior, sendo transferido para frascos erlenmeyer de $500 \mathrm{~mL}$ com água residuária sintética contendo fenol $\left(250 \mathrm{mg} \mathrm{L}^{-1}\right)$ e glicose (concentração conforme planejamento experimental), totalizando um volume de $150 \mathrm{~mL}$. A temperatura de todos os experimentos foi controlada a $25^{\circ} \mathrm{C}$; os ensaios estáticos $(0 \mathrm{rpm})$ foram conduzidos em estufa com circulação de ar e os experimentos realizados sob agitação (100 ou $200 \mathrm{rpm}$, conforme planejamento experimental) foram conduzidos em incubadora rotatória.

\section{Avaliação da tolerância ao fenol}

A partir da condição otimizada no planejamento experimental, foram realizados testes em triplicata para avaliar a tolerância do fungo filamentoso Aspergillus sp. LEBM2 na biodegradação em concentrações crescentes de fenol. Foram testadas concentrações acima de $500 \mathrm{mg} \mathrm{L}^{-1}$, que foi o limite de tolerância estabelecido anteriormente para este microrganismo quando cultivado em placas de Petri, tendo o fenol como única fonte de carbono. ${ }^{11}$

\section{Análise estatística}

Os dados obtidos foram tratados usando-se o software Statistica 5.0 (StatSoft, Inc.).

\section{RESULTADOS E DISCUSSÃO}

A Figura 1 mostra as médias das concentrações de fenol ao longo do tempo e os desvios padrão obtidos para a degradação deste

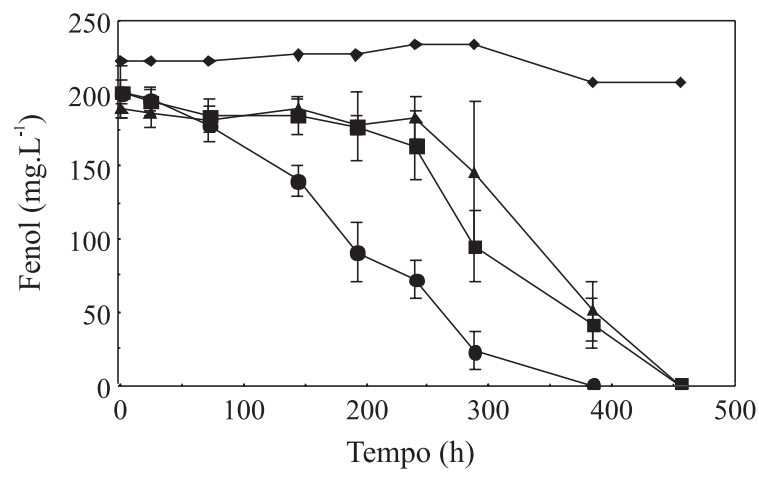

Figura 1. Ensaios de biodegradação de fenol por Aspergillus sp. LEBM2 em cultivo estático (sem agitação) utilizando diferentes substratos no preparo do inóculo. - fenol; • fenol + glicose; $\mathbf{\Delta}$ glicose; controle composto, utilizando-se diferentes substratos no preparo do inóculo. Mediante o ensaio controle verificou-se que não houve perdas abióticas significativas de fenol. A Figura 1 indica que a biodegradação foi mais rápida para o inóculo preparado com o substrato misto glicose e fenol, diminuindo o tempo para biodegradação total do fenol em 62 h. Comportamento similar foi observado por González et al. ${ }^{8}$ para a biodegradação de fenol por Pseudomonas putida.

Os resultados obtidos para taxa de biodegradação de fenol foram submetidos à análise de variância e comparação de médias utilizando $\mathrm{o}$ Teste de Tukey $(\mathrm{p}<0,05)$. A Figura 2 indica que a taxa de biodegradação obtida usando-se o inóculo preparado com o substrato misto glicose e fenol $\left(0,67 \pm 0,04 \mathrm{mg} \mathrm{L}^{-1} \mathrm{~h}^{-1}\right)$ foi estatisticamente superior e diferente dos demais ensaios. As taxas de biodegradação obtidas com os inóculos preparados somente com glicose $(0,44 \pm 0,04 \mathrm{mg}$ $\left.\mathrm{L}^{-1} \mathrm{~h}^{-1}\right)$ ou fenol $\left(0,41 \pm 0,02 \mathrm{mg} \mathrm{L}^{-1} \mathrm{~h}^{-1}\right)$ não apresentaram diferenças significativas entre si.

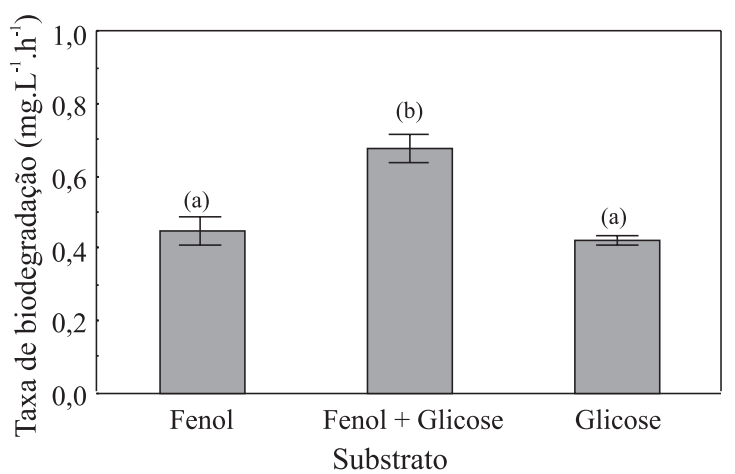

Figura 2. Taxas médias de biodegradação de fenol e \pm desvio padrão para os diferentes substratos utilizados no preparo do inóculo. Letras distintas indicam diferença significativa $(p<0,05)$

A partir desses resultados pôde-se constatar que a etapa de adaptação do microrganismo ao fenol durante o preparo do inóculo é essencial. A adaptação aumenta a taxa de biodegradação e a tolerância ao fenol, tornando o processo biológico mais eficiente. Por outro lado, também a presença de glicose como fonte adicional de carbono, facilmente assimilável, garante produção inicial de biomassa para a biodegradação.

Os resultados para o planejamento experimental $2^{3}$ são apresentados na Figura 3 e Tabela 1, sendo que a taxa de biodegradação de fenol variou de 0,58 a $3,76 \mathrm{mg} \mathrm{L}^{-1} \mathrm{~h}^{-1}$.

Os resultados obtidos foram ajustados a um modelo de primeira ordem (Equação 1) para a taxa de biodegradação de fenol (r) em função das variáveis significativas codificadas $\mathrm{X}_{1}$ (concentração de glicose) e $\mathrm{X}_{3}$ (velocidade de agitação).

$r=1,82+0,29 X_{1}+1,14 X_{3}+0,46 X_{1} X_{3}$

Com a análise de variância (ANOVA), apresentada na Tabela 2, onde as variáveis significativas foram acopladas ao resíduo, ${ }^{14}$ observase a validade do modelo pelo Teste $\mathrm{F}$, que foi 109,65 vezes maior que o valor tabelado, obtendo-se coeficiente de correlação de 0,995. A partir destes dados gerou-se a superfície de resposta e a curva de contorno apresentadas na Figura 4.

De acordo com a superfície de resposta (Figura 4a) e curva de contorno (Figura 4b), o incremento na agitação em toda a faixa de concentração de glicose avaliada proporcionou um aumento efetivo da taxa de biodegradação de fenol. Por outro lado, o aumento da concentração de glicose até agitação de $65 \mathrm{rpm}$ praticamente não influenciou a taxa de biodegradação. Porém, com agitações superiores 
(a)

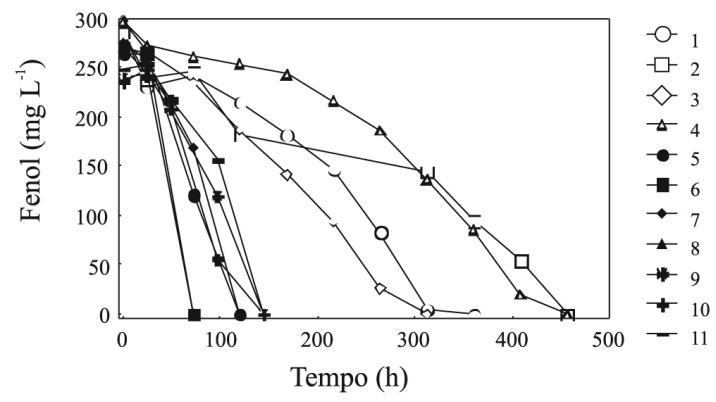

(b)

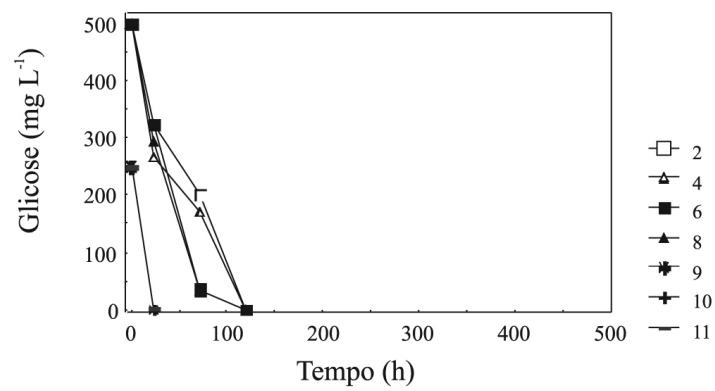

Figura 3. Ensaios de biodegradação de fenol propostos no planejamento experimental. (a) Concentração de fenol ao longo do tempo. (b) Concentração de glicose ao longo do tempo

Tabela 1. Planejamento fatorial completo $2^{3}$ em níveis codificados (valores reais)

\begin{tabular}{lcccccc}
\hline Ensaio & $\mathrm{X}_{1}$ & $\mathrm{X}_{2}$ & $\mathrm{X}_{3}$ & $\mathrm{r}_{\mathrm{exp}}$ & $\mathrm{r}_{\mathrm{pred}}$ & Desvio (\%) \\
\hline 1 & $-1(0)$ & $-1(10)$ & $-1(0)$ & 0,79 & 0,85 & $-7,60$ \\
2 & $+1(500)$ & $-1(10)$ & $-1(0)$ & 0,58 & 0,51 & 12,07 \\
3 & $-1(0)$ & $+1(20)$ & $-1(0)$ & 0,95 & 0,85 & 10,53 \\
4 & $+1(500)$ & $+1(20)$ & $-1(0)$ & 0,65 & 0,51 & 21,54 \\
5 & $-1(0)$ & $-1(10)$ & $+1(200)$ & 2,20 & 2,21 & $-0,46$ \\
6 & $+1(500)$ & $-1(10)$ & $+1(200)$ & 3,75 & 3,71 & 1,07 \\
7 & $-1(0)$ & $+1(20)$ & $+1(200)$ & 2,29 & 2,21 & 3,49 \\
8 & $+1(500)$ & $+1(20)$ & $+1(200)$ & 3,76 & 3,71 & 1,33 \\
9 & $0(250)$ & $0(15)$ & $0(100)$ & 1,66 & 1,82 & $-9,64$ \\
10 & $0(250)$ & $0(15)$ & $0(100)$ & 1,63 & 1,82 & $-11,66$ \\
11 & $0(250)$ & $0(15)$ & $0(100)$ & 1,72 & 1,82 & $-5,81$ \\
\hline
\end{tabular}

$\mathrm{X}_{1}=\operatorname{glicose}\left(\mathrm{mg} \mathrm{L}^{-1}\right) ; \mathrm{X}_{2}=$ volume de inóculo $(\%) ; \mathrm{X}_{3}=\operatorname{agitação~}(\mathrm{rpm})$; $r_{\exp }=$ taxa de biodegradação de fenol experimental $\left(\mathrm{mg} \mathrm{L}^{-1} \mathrm{~h}^{-1}\right) ; \mathrm{r}_{\text {pred }}=$ taxa de biodegradação de fenol predita pelo modelo $\left(\mathrm{mg} \mathrm{L}^{-1} \mathrm{~h}^{-1}\right)$.

Tabela 2. ANOVA para a taxa de biodegradação de fenol

\begin{tabular}{lcccc}
\hline Fonte de variação & $\begin{array}{c}\text { Graus de } \\
\text { liberdade }\end{array}$ & $\begin{array}{c}\text { Soma } \\
\text { quadrática }\end{array}$ & $\begin{array}{c}\text { Média } \\
\text { quadrática }\end{array}$ & Teste F \\
\hline Regressão & 3 & 12,879 & 4,293 & 477 \\
Resíduo & 7 & 0,064 & 0,009 & \\
Falta de ajuste & 5 & 0,060 & 0,012 & \\
Erro puro & 2 & 0,004 & & \\
Total & 10 & 12,944 & & \\
\hline
\end{tabular}

Coeficiente de correlação: 0,$995 ; \mathrm{F}_{0,95 ; 3 ; 7}=4,35$.

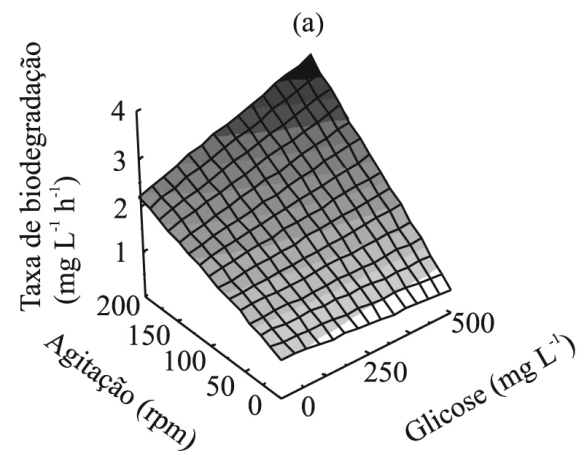

(b)

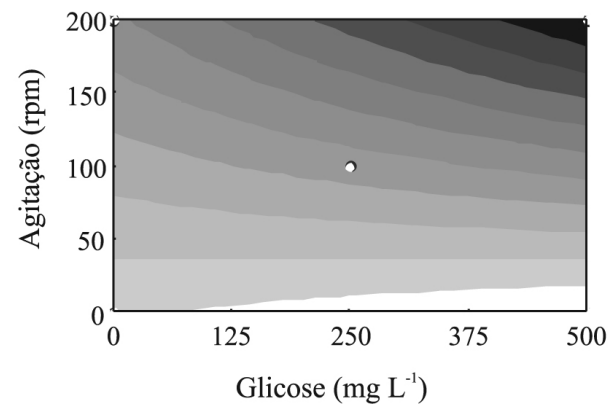

Figura 4. Superfície de resposta (a) e curva de contorno (b) para taxa de biodegradação de fenol em função da concentração de glicose e agitação

a $100 \mathrm{rpm}$, incrementando a concentração de glicose de 0 até 500 $\mathrm{mg} \mathrm{L}^{-1}$ registraram-se taxas de biodegradação de fenol de aproximadamente 0,8 a $3,5 \mathrm{mg} \mathrm{L}^{-1} \mathrm{~h}^{-1}$. Portanto, a agitação foi um parâmetro fundamental para o desempenho do Aspergillus sp. LEBM2 no processo de biodegradação.

Admitindo-se que haja uma correlação entre a velocidade de agitação do meio e o fornecimento de oxigênio proveniente do ar, o efeito positivo da agitação pode ser explicado por favorecer o metabolismo aeróbio do fungo, estimulando o crescimento microbiano e, desta forma, contribuindo para o sucesso da biodegradação.

Pelo acompanhamento do consumo de glicose e fenol nos ensaios do planejamento experimental (Figura 3) verificou-se que o fungo consumiu preferencialmente o carboidrato. Após o consumo da glicose, o fenol continuou sendo consumido, confirmando que o Aspergillus sp. LEBM2 pode utilizá-lo em seu metabolismo como única fonte de carbono.

O incremento da taxa de biodegradação de fenol ao utilizar-se maior concentração de glicose no meio explica-se devido à glicose ser um composto preferencialmente assimilado pelos fungos, favorecendo a formação de biomassa, e sua presença no substrato não implica que outros compostos não possam ser utilizados. Apesar da repressão catabólica ser freqüentemente mencionada, fontes de carbono convencionais, como glicose, glutamato e extrato de levedura, podem aumentar a taxa de biodegradação de compostos aromáticos, sugerindo que sua presença diminui a toxicidade e o efeito inibitório sobre o crescimento celular exercido por compostos xenobióticos. ${ }^{15}$ Desta forma, esta habilidade de degradar fenol mesmo na presença de glicose implica na capacidade que o fungo tem de remover fenol em efluentes, solos e aquíferos que contenham outras fontes de carbono mais facilmente assimiláveis.

Analisando a superfície de resposta (Figura 4a), a condição otimizada para minimização do tempo de biodegradação foi $500 \mathrm{mg}$ $\mathrm{L}^{-1}$ de glicose e $200 \mathrm{rpm}$ de agitação, independente do volume de inóculo, conseguindo-se aumento de 6,28 vezes na taxa de biodegradação (ensaios 6 e 8) quando comparada ao ensaio menos eficiente. 
Sugere-se que seja utilizado volume de inóculo de 20\% (ensaio 8), para garantir a eficiência do processo para maiores concentrações de fenol, uma vez que se trata de uma substância tóxica. Na Tabela 1 observa-se ainda que o modelo proposto prevê muito bem a taxa de biodegradação de fenol, principalmente na região ótima, onde apresenta desvios relativos inferiores a $2 \%$.

A Figura 5a apresenta os resultados obtidos para a biodegradação de fenol utilizando diferentes concentrações deste componente, permitindo afirmar que houve aumento no tempo de biodegradação conforme incremento na concentração de fenol.

(a)

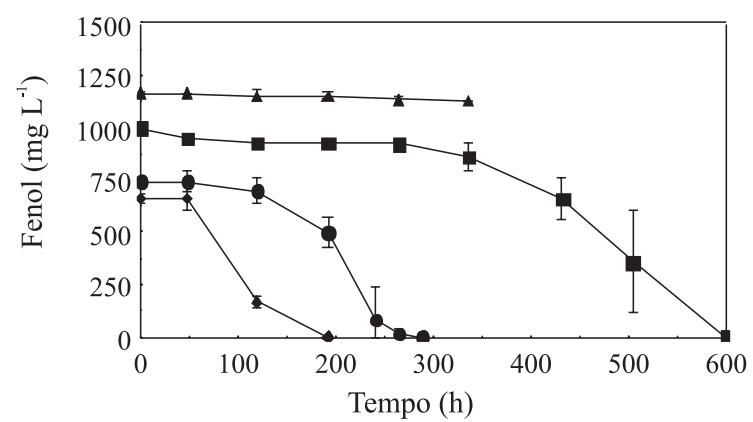

(b)

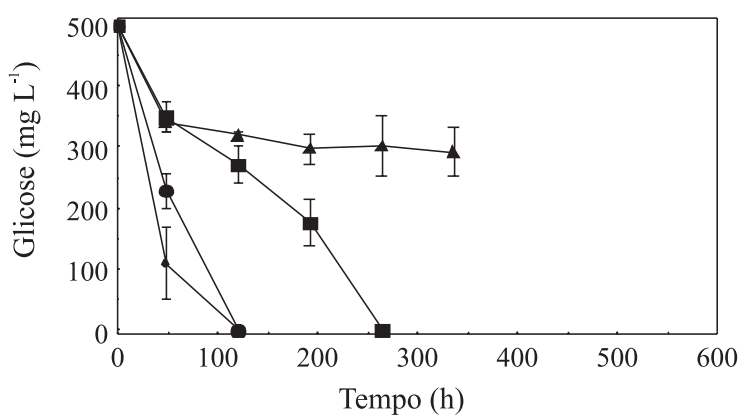

Figura 5. Consumo de fenol (a) e glicose (b) para diferentes concentrações iniciais de fenol, nas condições estabelecidas pelo planejamento experimental (500 $\mathrm{mg} \mathrm{L}^{-1}$ de glicose, $200 \mathrm{rpm}$ de agitação, $20 \%$ de inóculo). $663 \mathrm{mg} \mathrm{L}^{-1}$; - $743 \mathrm{mg} \mathrm{L}^{-1}$; - $989 \mathrm{mg} \mathrm{L}^{-1}$; $1166 \mathrm{mg} \mathrm{L}^{-1}$

A biodegradação foi total até a concentração inicial de fenol de $989 \pm 15 \mathrm{mg} \mathrm{L}^{-1}$. Até esta concentração inicial, o fungo consumiu a glicose preferencialmente, mas simultaneamente ao fenol, para após consumir totalmente o fenol (Figuras 5 a e 5b). Observa-se também na Figura 5b que, aumentando a concentração de fenol, a glicose foi consumida mais lentamente, demonstrando claramente o efeito inibidor do fenol sobre o metabolismo do fungo.

Como pode ser observado na Figura 6, não foi verificada diferença significativa na taxa de biodegradação de fenol entre as concentrações iniciais de $663 \pm 20$ e $743 \pm 22 \mathrm{mg} \mathrm{L}^{-1}$. A concentração de $989 \pm 15$ $\mathrm{mg} \mathrm{L}^{-1}$ foi diferente das demais, apresentando taxa de biodegradação de fenol inferior $\left(1,66 \pm 0,02 \mathrm{mg} \mathrm{L}^{-1} \mathrm{~h}^{-1}\right)$, cerca de $50 \%$ menor que as anteriores, provavelmente por estar se aproximando do limite de tolerância ao fenol. A menor taxa de biodegradação mostrou-se associada a um aumento da fase de adaptação, evidenciada pela pequena variação na concentração de fenol nas primeiras 264 h de cultivo.

Foi observada uma variação não expressiva na concentração de fenol, cerca de $4 \%$, para a concentração inicial de $1166 \pm 11 \mathrm{mg} \mathrm{L}^{-1}$ durante o período acompanhado, apesar do consumo de glicose nas primeiras $192 \mathrm{~h}$ de cultivo. A partir deste ponto também não houve consumo de glicose. Este fato indica que nesta concentração inicial de fenol o fungo provavelmente permaneceu viável pelo período

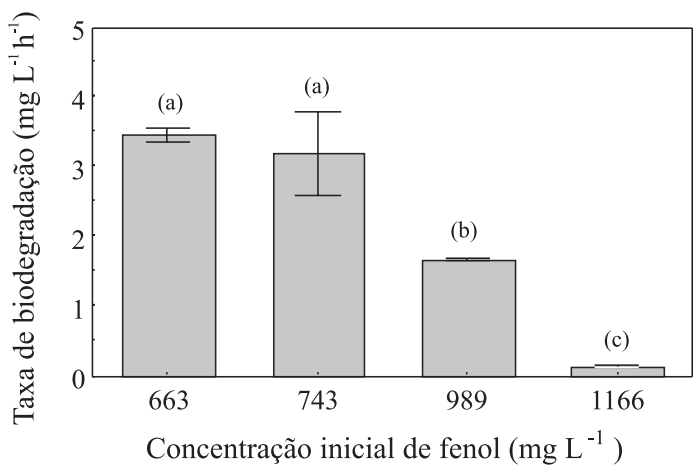

Figura 6. Taxas médias de biodegradação de fenol para diferentes concentrações iniciais e \pm desvio padrão. Letras distintas indicam diferença significativa $(p<0,05)$

citado, sendo que após sua atividade metabólica cessou pelo efeito tóxico do fenol, pois não mais se verificou decréscimo na concentração de glicose.

A concentração inicial de $989 \pm 15 \mathrm{mg} \mathrm{L}^{-1}$ pode ser considerada elevada tratando-se de fenol, o que possibilita afirmar que Aspergillus sp. LEBM2 apresenta alta tolerância ao fenol, sendo capaz de biodegradá-lo totalmente mesmo em altas concentrações. Limites de tolerância ao fenol em níveis similares têm sido observados para outros microrganismos cultivados em batelada, como Candida tropicalis, ${ }^{7}$ Pseudomonas putida ${ }^{8}$ e Acinetobacter sp.. ${ }^{9}$ Um linhagem de Candida tropicalis ${ }^{3}$ foi capaz de degradar até $2000 \mathrm{mg} \mathrm{L}^{-1}$. Graphium sp. ${ }^{6}$ foi capaz de tolerar $2000 \mathrm{mg} \mathrm{L}^{-1}$, mas a degradação foi de aproximadamente $60 \%$. Para o gênero Aspergillus, Santos e Linardi ${ }^{1}$ observaram percentuais de biodegradação variando de cerca de $90 \%$ (concentração inicial até $\left.600 \mathrm{mg} \mathrm{L}^{-1}\right)$ até $4 \%$ (1000 $\left.\mathrm{mg} \mathrm{L}^{-1}\right)$, com taxas de biodegradação na faixa de 3 a $4 \mathrm{mg} \mathrm{L}^{-1} \mathrm{~h}^{-1}$.

Desta forma, é importante destacar que com Aspergillus sp. LEBM2 foi possível a biodegradação total em altas concentrações de fenol, apesar do decréscimo na taxa de biodegradação.

\section{CONCLUSÕES}

Constatou-se que a adaptação prévia do fungo Aspergillus sp. LEBM2 em meio contendo fenol e glicose, ambos na concentração de $250 \mathrm{mg} \mathrm{L}^{-1}$, possibilitou maior taxa de biodegradação de fenol, tornando o processo mais eficiente. Os ensaios em frascos agitados apresentaram resultados promissores, permitindo otimizar algumas variáveis que incrementaram a taxa de biodegradação de fenol em até 6,48 vezes. As condições otimizadas para alcançar uma taxa de biodegradação de fenol de $3,76 \mathrm{mg} \mathrm{L}^{-1} \mathrm{~h}^{-1}$ foram $500 \mathrm{mg} \mathrm{L}^{-1}$ de glicose e agitação de $200 \mathrm{rpm}$, sugerindo-se que seja utilizado um volume de inóculo de $20 \%$ para garantir a eficiência do tratamento para maiores concentrações de fenol. Aspergillus sp. LEBM2 tem alta tolerância ao fenol, podendo biodegradá-lo efetivamente até uma concentração de $989 \pm 15 \mathrm{mg} \mathrm{L}^{-1}$ apresentando, desta forma, grande potencial para emprego em processos de bioaumentação.

\section{AGRADECIMENTOS}

Ao apoio financeiro do Conselho Nacional de Desenvolvimento Científico e Tecnológico - CNPq, o qual possibilitou a realização desse trabalho.

\section{REFERÊNCIAS}

1. Santos, V. L.; Linardi, V. R.; Process Biochem. 2004, 39, 1001. 
2. Sancinetti, S. T.; Félix, J. P. L.; Neto, M. A. F.; Anais do XIV Simpósio Nacional de Bioprocessos, Florianópolis, Brasil, 2003.

3. Britto, J. M.; Rangel, M. C.; Quim. Nova 2008, 31, 114.

4. García, I. G.; Peña, P. R. J.; Venceslada, J. L. B.; Martín, A. M.; Santos, M. A. M.; Gómez, E. R.; Process Biochem. 2000, 35, 751.

5. Santos, V. L.; Heilbuth, N. M.; Braga, D. T.; Monteiro, A. S.; Linardi, V. R.; J. Basic Microbiol. 2003, 43, 238.

6. Yan, J.; Jianping, W.; Hangmei, L.; Soliang, Y.; Tongding, H.; Biochem. Eng. J. 2005, 24, 243.

7. Chen, K. C.; Lin, Y. H.; Chen, W. H.; Liu, Y. C.; Enzyme Microb. Technol. 2002, 31, 490.

8. González, G.; Herrera, G.; García, M. T.; Peña, M.; Bioresour. Technol. 2001, 80,137
9. Adav, S. S.; Chen, M. Y.; Lee, D. J.; Ren, N.Q.; Chemosphere 2007, 67, 1566.

10. Hidalgo, A.; Jaureguibeitia, A.; Prieto, M. B.; Rodríguez-Fernández, C.; Serra, J. L.; Llama, M. J.; Enzyme Microb. Technol. 2002, 31, 221.

11. Santos, E. O.; Rosa, C. F. C.; Passos, C. T.; Sanzo, A. V. L.; Burkert, J. F. M.; Kalil, S. J.; Burkert, C. A. V.; Afr. J. Biotechnol. 2008, 7, 1314.

12. Burkert, J. F. M.; Tese de Doutorado, Universidade Estadual de Campinas, Brasil, 2003.

13. Miller, G. L.; Anal. Chem. 1959, 31, 426.

14. Rodrigues, M. I.; Iemma, A. F.; Planejamento de Experimentos e Otimização de Processos: uma estratégia seqüencial de planejamentos, Casa do Pão Editora: Campinas, 2005

15. Loh, K. C.; Wang, S. J.; Biodegradation 1998, 8, 329. 